\title{
A male pheromone-mediated trade-off between female preferences for genetic compatibility and sexual attractiveness in rats
}

Yao-Hua Zhang and Jian-Xu Zhang*

\begin{abstract}
Introduction: Chemosensory signals play a vital role in socio-sexual interactions of rodents. Females rely heavily on chemosensory signals to evaluate genetic similarity and quality of potential mates, but their olfactory preferences for these criteria often conflict in mate choice.

Results: Using two inbred strains of rats, Brown Norway (BB) and Lewis (LL) and their F1 reciprocal hybrids (BL, BBo

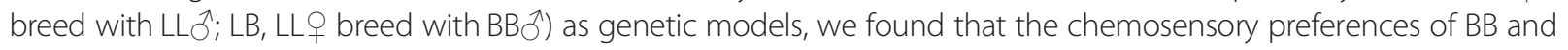
LL females between these 4 strains of rats could be predicted on the basis of genetic compatibility benefits, except that $L L$ females exhibited incestuous preferences for male urine odor of $L L$ rats over that of the BB strain and the F1 hybrids. Seven ketone components of major urine volatiles proved to be potential male pheromones and were enriched in LL males compared to BB males or the F1 hybrid males. We hypothesize that these ketones produced an extravagant male trait that attracts LL females, overriding compatibility traits. This conclusion was corroborated by adding three synthetic pheromone analogues, 4-heptanone, 2-heptanone and 9-hydroxy-2-nonanone of these 7 components, which resulted in equalization of the sexual attractiveness of BB male urine and LL male urine. Additionally,

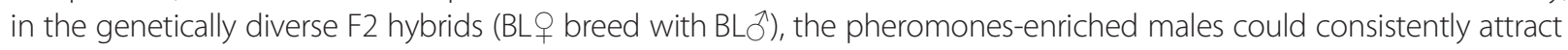
the F2 females.
\end{abstract}

Conclusions: We suggest that the exaggerated male pheromones serve as a "sexual chemical ornament" to attract females, independent of genetic compatibility, whereas genetic dissimilarity could influence the preferences only when male pheromones varied on a small scale.

Keywords: Compatibility, Male pheromones, Olfactory preference, Quality

\section{Introduction}

In rodents, male urine odor can signal genetic relatedness, based on genotype-specific odortype and genetic quality indicated by sexually selected signals for female mate assessment and choice [1-5]. However, mating preferences for compatibility are distinguished from mating preferences for sexual attractiveness: compatibility depends on the genotypic matching between males and females, whereas sexually attractive traits are uniformly favored by all the potential mates $[4,6,7]$. Females prefer genetically dissimilar males and/or males whose sexually selected signals indicate high genetic quality; however, a sexually

\footnotetext{
* Correspondence: zhangjx@ioz.ac.cn

State Key Laboratory of Integrated Management of Pest Insects and Rodents in Agriculture, Institute of Zoology, Chinese Academy of Sciences, 1\# Bei-Chen West Road, Beijing 100101, China
}

attractive partner is not necessarily the compatible one and vice versa, so mating preferences for compatibility and sexual attractiveness criteria are often in conflict [4]. However, the way in which male pheromone molecules act as sexually selected signals to interact with genotype-correlated odorant signals in determining female mate choice decision has seldom been tested empirically $[1,4,5,7-10]$.

Several hypotheses have been put forward to explain the evolutionary process of mate choice to obtain indirect genetic benefits, such as good genes, sexy son and heterozygous benefits [11]. The nocturnal rodents have a well-developed sense of smell; female mate choice mainly depends on male odorant signals, which contain information both about genetic compatibility and genetic quality (indicating the possibility of "good genes" and 
sexy son benefits) $[4,6,11,12]$. Avoidance of incestuous mating is widely reported across animal taxa, where the major histocompatibility complex (MHC) has provided an excellent example to illustrate the genetic compatibilitybased mate choice mechanism [4,12-15]. However, theory predicts that an individual that mates with a relative will spread genes identical by descent, which also offers a positive effect on the inclusive fitness of parents $[16,17]$. In some animal species, for instance, the fruit fly (Drosophila melanogaster), the cestode (Schistocephalus solidus) and the cichlid fish (Pelvicachromis taeniatus), the females display significant preferences for mating with their brothers over unrelated males [18-21]. In natural populations of animals, mating among relatives occurs more often than expected by chance, suggesting that genetic compatibility effects are limited [6,22].

Almost all research on odor-based mate choice in mice (Mus musculus) and rats (Rattus norvegicus) have shown that females chose genetically compatible mates to obtain heterozygous offspring and high reproductive success $[1,2,4,5,13,14,23]$. Exceptionally, Roberts and Gosling [4] found that "compatible genes" and "good genes" interact in female mate choice in mice, and their relative influence can vary with the degree of variability of each trait among available males. In rodents, both genetic compatibility and male sexual attractiveness can be signaled by urinary volatiles derived from bladder urine (BU) and preputial gland secretion (PGS) and major urinary protein (MUP) from liver; however, the ways in which genetic relatedness- and qualityrelated signaling molecules interact in female mate choice has not been tested experimentally $[1,4,5,8-11,24,25]$.

Numerous inbred strains of laboratory mice and rats provide ideal animal models to study odor-based mate choice $[4,13,23]$. Individuals of an inbred strain are nearly genetically identical to each other (like identical twins), but genetically distinct across different inbred strains $[26,27]$. The reciprocal hybrids share the same complement of genes, and have intermediate relatedness between the two parental strains. Previous work in our laboratory using two inbred strains of rats, Lewis (LL) and Brown Norway (BB), showed that BB females preferred the male urine odor of LL male to that of their own strain [5]. Here, unlike BB females, LL females display an olfactory preference for the males of their own strain over $\mathrm{BB}$ males, violating genetic compatibility benefits. We use these inbred strains, their reciprocal hybrids $\left(\mathrm{BL}, \mathrm{BB}_{+} \times \mathrm{LL}^{\uparrow} ; \mathrm{LB}, \mathrm{LL}_{+} \times \mathrm{BB}^{\lambda}\right)$ and $\mathrm{BL} \mathrm{F} 2$ hybrids $\left(\mathrm{BL}_{+} \times \mathrm{BL}^{\hat{\lambda}}\right)$ as genetic models to examine whether advantages of mating with close kin override the effects of inbreeding depression, and how the male sex pheromone mediates the trade-off between preferences for genetic compatibility and genetic quality in female mate choices.

\section{Results}

Urine scent-based preference test by BB and LL females

In binary choice tests, $\mathrm{BB}$ females showed an olfactory preference for LL male urine over that of the other three strains (BB vs. LL: $P=0.032, T=2.455, N=12$; LL vs. LB: $P=0.021, T=2.688, N=12$; LL vs. BL: $P=0.015, Z=$ 2.433, $N=12$ ). BB females preferred BL male urine odor to that of $\mathrm{BB}$ males, but showed no preference between either BB or BL and LB male urine (BB vs. BL: $P<0.001$, $T=7.097, N=12$ ) (Figure 1A).

On the other hand, LL females displayed an incestuous preference for male urine odor of their own strain to that of the other three strains (LL vs. BB: $P=0.009$, $Z=2.621, N=13$; LL vs. LB: $P=0.039, T=2.320, N=12$; LL vs. BL: $P=0.001, T=3.180, N=13$ ); however, they preferred male urine odor of the $\mathrm{BB}$ strain compared to that of the genetically more similar $\mathrm{LB}$ and $\mathrm{BL}$ strains $(\mathrm{BB}$ vs LB: $P=0.005, Z=2.830, N=13$; BB vs. $B L: P=0.002$, $Z=3.040, N=13)$. In addition, LL females showed no preference between LB and BL male urine odor (Figure 1B).

In these rats, within-strain breeding resulted in inbreeding depression such as reduced reproductive successes and offspring (Additional file 1: Table S1).

\section{The urine-borne volatile profiles varying among strains}

Thirty BU volatiles and 24 PGS volatiles were detected by GC-MS (Additional file 2: Figure S1 and Additional file 3: Figure S2). The relative abundances (percentage of summed peak area) of these volatiles were subjected to a principle component analysis (PCA). In the case of BU volatiles, $\mathrm{BB}$ males were distinct from LL males but the hybrids were poorly separated from LL (Additional file 4: Figure S3A). In the case of PGS volatiles, BB males were nearly separated from LL, and the two hybrids clustered together (Additional file 5: Figure S4A). ANOVA with a post hoc LSD test revealed that $16 \mathrm{BU}$ and 10 PGS volatile components were different in relative abundances between BB and LL male rats (Additional file 1: Table S2 and Table S3). Although the 4 strains were not clearly separated in the PCA plots, BB had 14 BU and 10 PGS volatiles that differed from LB, and 15 BU and 5 PGS volatiles that differed from BL. LL had 3 BU and 11 PGS volatiles that differed from $\mathrm{LB}$, and 7 BU and 10 PGS volatiles that differed from BL. LB and BL hybrid males clustered together in the PCA plots, and neither BU nor PGS compounds varied in relative abundance (Additional file 1: Table S2 and Table S3; Additional files 4 and 5: Figure S3A and Figure S4A).

Regarding the abundances (peak areas in GC graphs) of these volatiles, PCA revealed that, in the case of BU, $\mathrm{BB}$ and LL males were clearly separated, but they were poorly separated from hybrid males (Additional file 4: Figure S3B). In the case of PGS, LL males were nearly separated from the other three strains, whereas the 


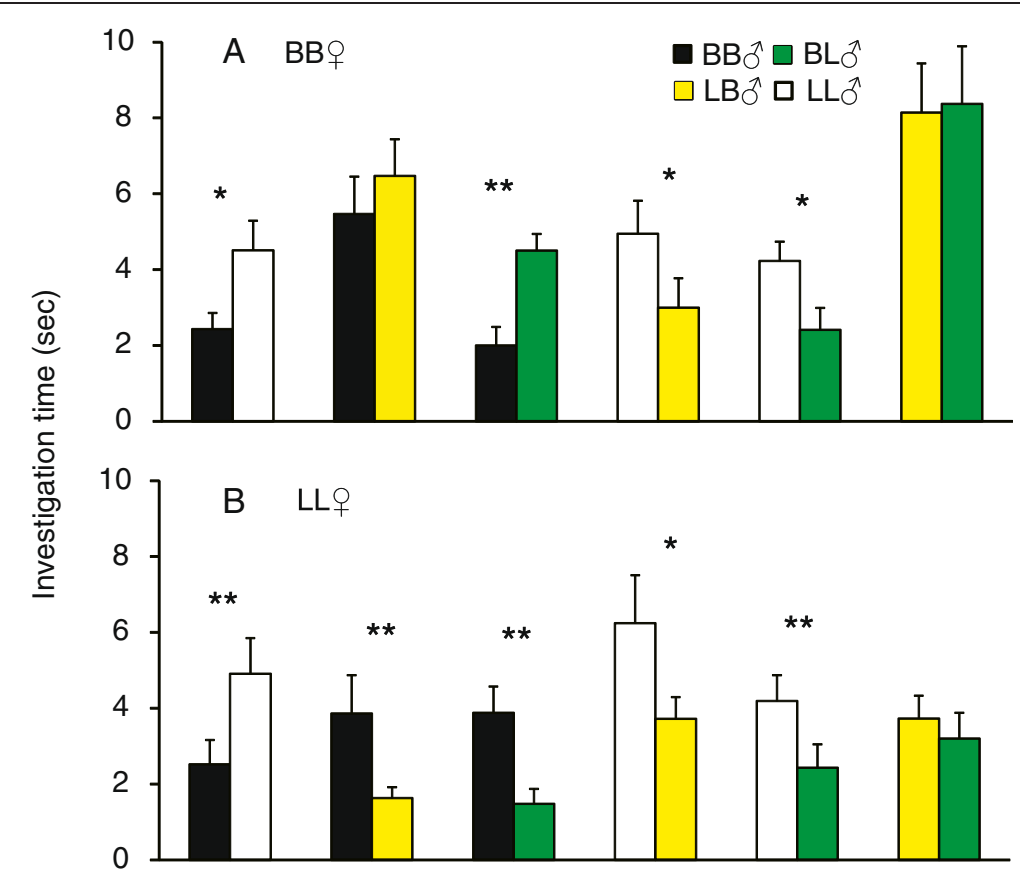

Figure 1 Duration of investigation (mean $\pm \mathrm{SE})$ by $\mathrm{BB}(\mathrm{A}, N=12)$ and $\mathrm{LL}(\mathrm{B}, N=13)$ female rats in binary choice tests $\left({ }^{*} P<0.05,{ }^{* *} P<0.01\right.$, paired $t$-test or Wilcoxon signed-rank test).

hybrids and $\mathrm{BB}$ males were not separated (Additional file 5: Figure S4B). ANOVA with a post hoc LSD test revealed that $15 \mathrm{BU}$ and 8 PGS volatile components were different in abundances between BB and LL male rats (Additional file 1: Table S4 and Table S5). Although the 4 strains were not clearly separated in the PCA plots, BB had 8 BU and 5 PGS volatiles that differed from LB, and 6 BU and 3 PGS that differed from BL. LL had $9 \mathrm{BU}$ and 10 PGS volatiles that differed from $\mathrm{LB}$, and $15 \mathrm{BU}$ and 8 PGS volatiles that differed from BL. LB and BL hybrid males clustered together in the PCA plots, and neither BU nor PGS compounds varied in relative abundance (Additional file 1: Table S4 and Table S5; Additional files 4 and 5: Figure S3B and Figure S4B).

\section{The volatiles exaggerated in LL male urine and potential} male pheromones

Of the $16 \mathrm{BU}$ volatile components varying between the rat strains, 7 (Peaks: 2, 3, 11, 15, 17, 19 and 22) had a relative abundance $>1 \%$ and, together accounted for $83.51 \%$ of all the 16 compounds. These 7 compounds were male-biased in BB, LL and the hybrids and had consistently higher relative abundance or absolute abundance in LL males than in BB and hybrid males (Figure 2, Additional file 6: Figure S5, Additional file 1: Table S2 and Table S4; and data not shown). However, the 14 malebiased PGS compounds showed inconsistent differences between rat strains (Additional file 1: Table S3 and Table $\mathrm{S} 5)$. These volatiles in $\mathrm{LB}$ and $\mathrm{BL}$ males of the hybrids from reciprocal crosses between $\mathrm{BB}$ and LL rats seemed to have intermediate levels (Figure 2).

Furthermore, the above-mentioned 7 BU volatiles were severely suppressed in absolute abundances (Figure 3A) or in relative abundances (Figure 3B) by orchiectomy and restored by testosterone-treatment in LL male rats (Figure 3, Additional file 1: Tables S6 and Additional file 7: Figure S6).

Meanwhile, LL females were used as odor recipients. When synthetic analogs of 4-heptanone $(4 \mathrm{H}), 2$-heptanone $(2 \mathrm{H})$ and 9-hydroxy-2-nonanone $(9 \mathrm{H} 2 \mathrm{~N})$ selected from the 7 compounds were replenished into castrated male urine so that levels were equal to those of $\mathrm{LL}$ males $(4 \mathrm{H}=$ $1.5 \mathrm{ppm} ; 2 \mathrm{H}=22 \mathrm{ppm} ; 9 \mathrm{H} 2 \mathrm{~N}=7 \mathrm{ppm}$ ), the sexual attractiveness of castrated male urine were restored (sham vs. castrated: $P=0.004, T=3.513, N=14$; castrated vs. castrated + the 3 ketones: $P=0.041, Z=2.040, N=14$; sham vs. castrated + the 3 ketones: $P=0.926, T=0.095, N=14$ ) (Figure 4, Additional file 1: Tables S6).

\section{Male pheromones and LL female preferences}

We further supplemented the the above-mentioned 3 ketones into $\mathrm{BB}$ male urine, which greatly boosted the attractiveness of $\mathrm{BB}$ male urine to LL females, especially when the 3 ketones reached $75 \%$ and $100 \%$ of the LL levels $(4 \mathrm{H}=0.06 \mathrm{ppm}, 2 \mathrm{H}=3.76 \mathrm{ppm}, 9 \mathrm{H} 2 \mathrm{~N}=0.86 \mathrm{ppm}$; $\mathrm{BB}$ vs. $\mathrm{BB}+75 \% 3$ ketones, $P=0.008, Z=2.669, N=14$; $\mathrm{BB}$ vs. $\mathrm{BB}+100 \% 3$ ketones, $P=0.016, \quad T=2.769, \quad N=14$; Figure $5 \mathrm{~A})$. At same time, the attractiveness of these 

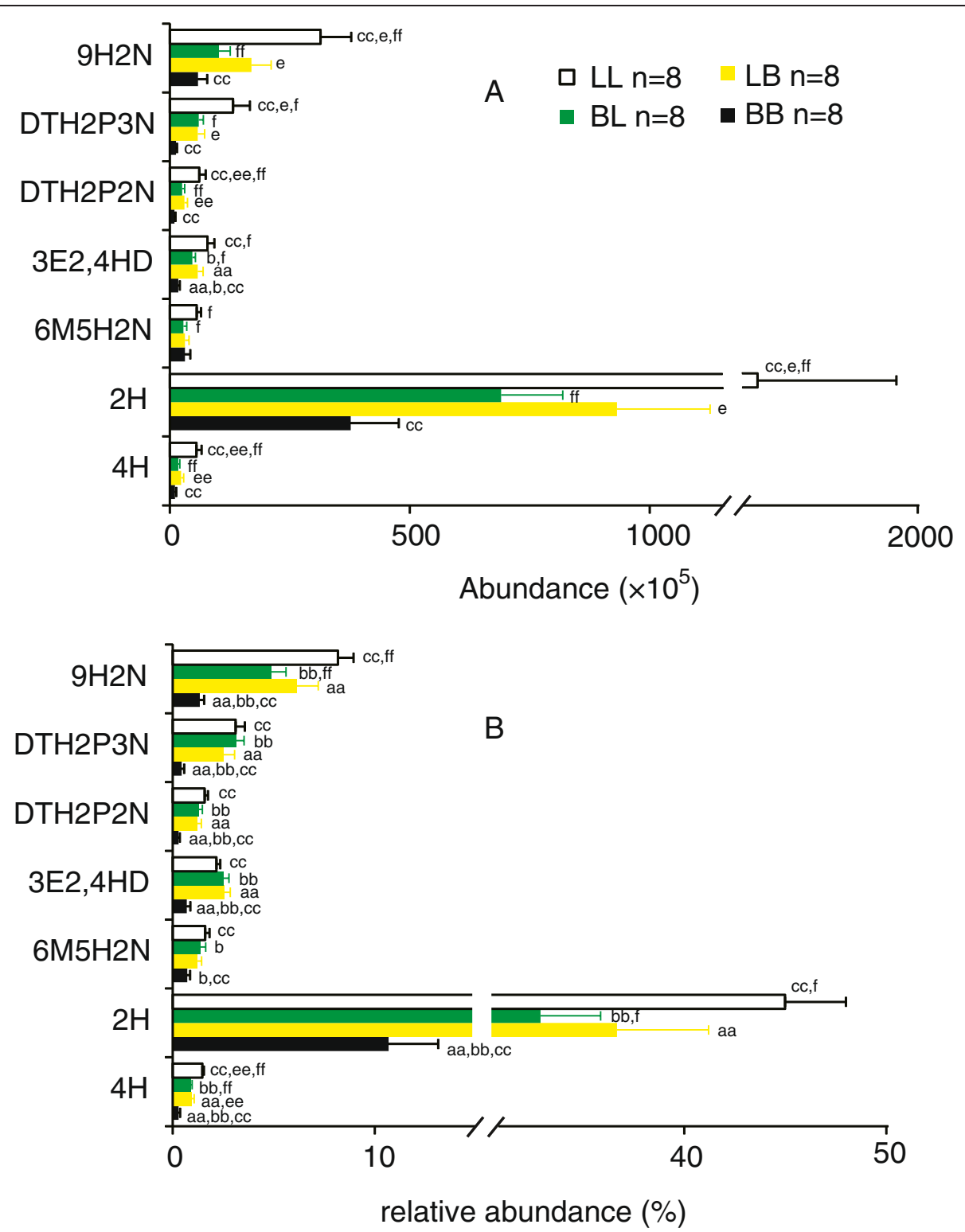

Figure 2 Comparison of 7 urinary major ketones between inbred and hybrid males: abundances $(A)$, relative abundances $(B)(N=8$, mean $\pm S E$, one way ANOVA with post hoc LSD test, the same superscript letters indicate significant difference, single letter, $P<0.05$; double letters, $\mathbf{P}<\mathbf{0 . 0 1}$. 4H: 4-Heptanone, 2H: 2-Heptanone, 6M5H2N: 6-Methyl- 5-hepten-2-one, 3E2,4HD: 3-Ethyl-2,4-heptanedione, DTHP2N: a dialkyl tetrahydro-2H-pyran-2-one, 9H2N: 9-hydroxy-2-Nonanone).

treated $\mathrm{BB}$ urine was comparable to that of LL male urine (LL vs. $\mathrm{BB}+75 \% 3$ ketones, $P=0.102, T=1.761, N=14$; LL vs. $\mathrm{BB}+100 \% 3$ ketones, $N=14, P=0.265, T=1.164$, $N=14 ;$ Figure $5 \mathrm{~B})$.

\section{Male pheromones and female preferences in $\mathrm{F} 2$ hybrids} In F2 hybrids crossed from F1 hybrids $(\mathrm{BL}+\times \mathrm{BL} \hat{\jmath})$, F2 females also preferred male urine scent of LL rats to that of $\mathrm{BB}$ rats $(P<0.001, T=5.403, N=40$; Figure $6 \mathrm{~A})$. The three groups we selected from F2 males showed significant differences in their ketone levels (Figure 6B). F2 females exhibited a significant preference for male urine scent of
HIGH group to that of LOW group (HIGH vs. LOW, $P=0.009, Z=2.621, N=40$ ), but showed no preference between other groups (MEDIUM vs. LOW, $P=0.989$, $Z=0.013 ;$ MEDIUM vs. HIGH, $P=0.129, \quad Z=1.519$; $N=40$; Figure 6C).

\section{Discussion}

In binary choice tests, BB females exhibited an olfactory preference for more genetically dissimilar males, in most cases as expected from the genetic compatibility hypothesis [11]. Although LL females also showed preferences for compatible benefits between $\mathrm{BB}$ and the F1 hybrid male 

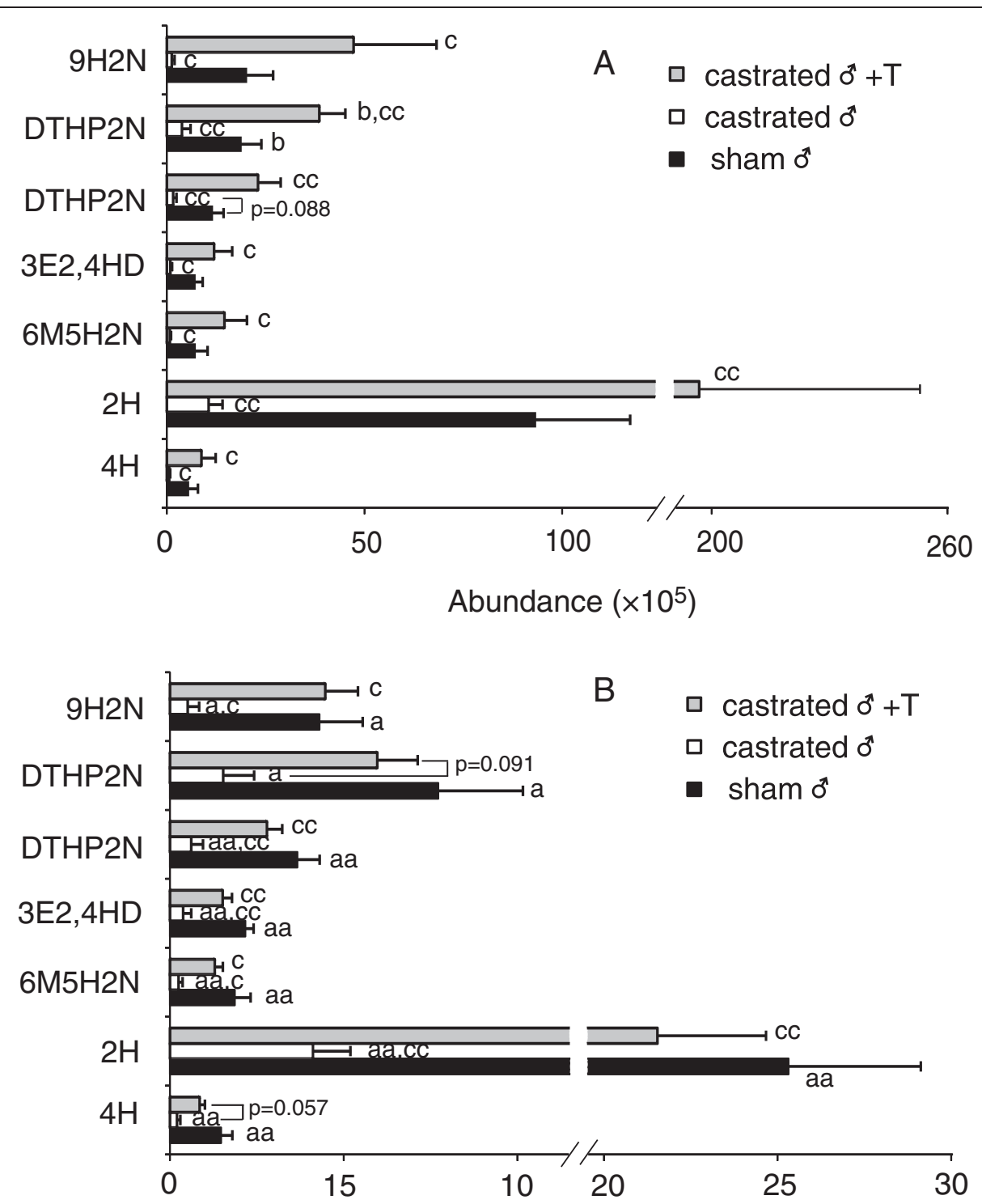

Relative abundance (\%)

Figure 3 Comparison of 7 urinary major ketones between male rats with different hormone status: abundances (A), relative abundances $(B)(N=8$, mean $\pm S E$, one way ANOVA with post hoc LSD test, the same superscript letters indicate significant difference, single letter, $P<\mathbf{0 . 0 5}$; double letters, $P<\mathbf{0 . 0 1}$. 4H: 4-Heptanone, 2H: 2-Heptanone, 6M5H2N: 6-Methyl- 5-hepten-2-one, 3E2,4HD: 3-Ethyl-2,

4-heptanedione, DTHP2N: a dialkyl tetrahydro-2H-pyran-2-one, 9H2N: 9-hydroxy-2-Nonanone).

rats, they chose male urine of genetically identical LL rats over that of $\mathrm{BB}$ or the F1 hybrid rats at the cost of inbreeding. Male pheromone levels might be positively correlated with genetic quality in male animals, and thus signal the information to choosy females about genetic benefits, such as "good genes" and sexy son benefits $[4,5,11,13,15,23,26,28]$. Preferences for genetic compatibility strictly depend on the interplay of the genotypes of potential partners and are different from directional preferences for ornamental secondary sexual traits $[4,7]$. Thus, the most compatible partner may not be the one with most pronounced ornaments and vice versa [4,6,7]. Genetic compatibility criteria and quality criteria may often conflict, and are often difficult to reconcile with female mate choice $[4,6,7]$. In mice, genetic quality indicated by urine marking rates largely determine female mate choice, whereas genetic compatibility works only when variability in genetic dissimilarity among the males is relatively large, or conversely, when the variability in marking rates is small [4]. However, in the cichlid fish, females weighed genetic compatibility of male mates stronger than quality traits (body size) [7]. Here, the contents of male pheromone 

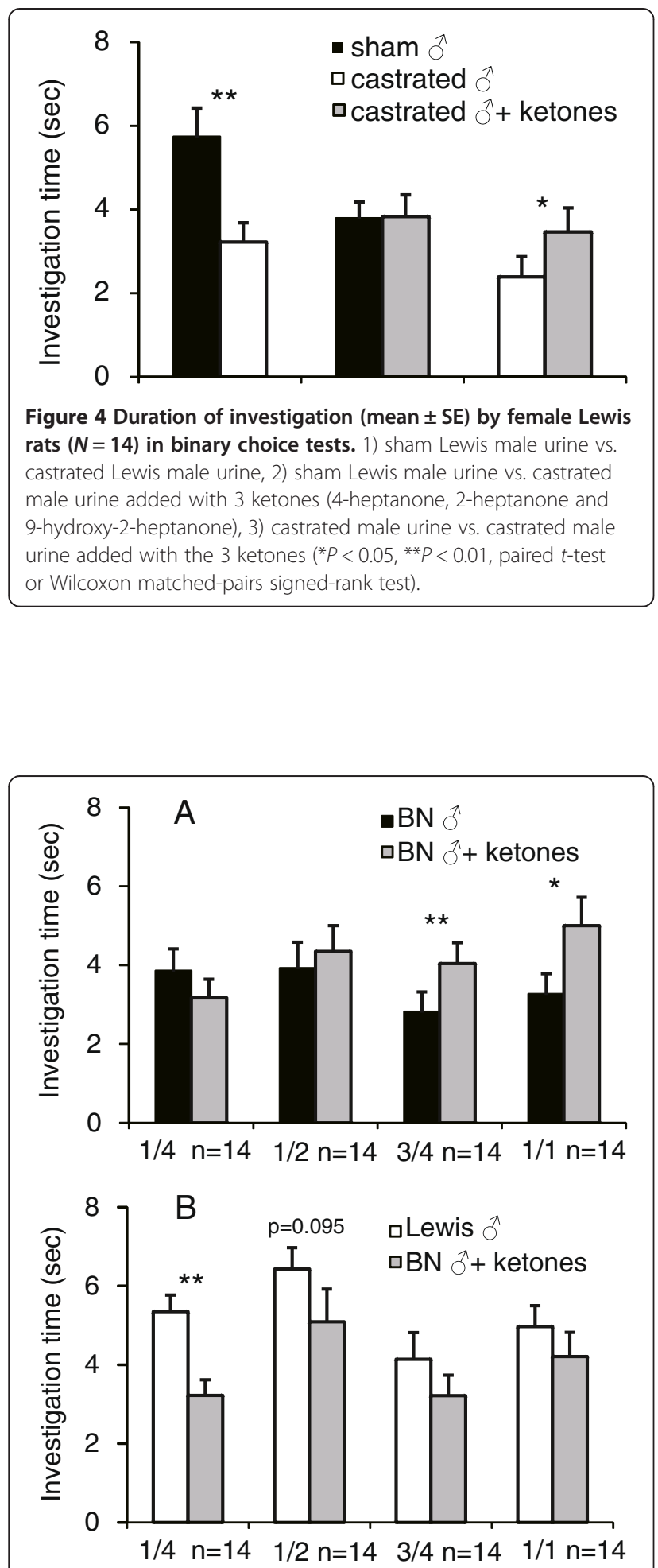

Figure 5 Duration of investigation (mean \pm SE) by female LL rats $(N=13)$ of different male urine samples during a 3-min binary choice test. Ketones were added into BB male urine, and their levels were equal to $1 / 4,1 / 2,3 / 4,1$ of those in $L L$ males. (A) BB male urine vs. $B B$ male urine added with ketones, (B) $L L$ male urine vs. BB male urine added with ketones $\left({ }^{*} P<0.05,{ }^{*} P<0.01\right.$, paired t-test or Wilcoxon signed rank test). components quantitatively varied among the 4 strains: LL males were highest, $\mathrm{BB}$ males were lowest and the hybrid males were intermediate. Coincidently, LL female displayed incestuous preferences only between LL and the others. At the same time, when increasing the urinary ketones of $\mathrm{BB}$ male urine to $75 \%$ and $100 \%$ of those in that of LL, the treated BB males could be as attractive as LL. We therefore inferred that the genetic compatibility worked only when the sexual chemical ornaments were below the threshold. Above the threshold, the influence of a sexy trait would override the compatibility benefits.

In the binary choice test, the paired male urine scent of different strains, except the BL-LB hybrid pairs, received different responses by female LL or BB rats, and therefore must contain strain- or genotype-specific odorant signals to advertise genetic similarity and quality to females $[1,2,4,5,9,13]$. The chemosignals that provide olfactory information about individuality, strain and species are often composed of a large number of compounds in a mosaic manner or a few key components that vary in relative abundance [1,5,29-31]. In the current study, LL male urine might also contain some chemosignals, which might be analogous to some extravagant ornamental traits in birds and fishes and related to sexual attractiveness and high quality, and thus incur an inbreeding preference that overrides inbreeding avoidance by LL females $[4,11,15,32,33]$. Such conjectures are confirmed by the following chemical analysis of urine-borne volatiles.

In rats, the voided urine contains volatile compounds excreted from both bladder urine and preputial glands $[5,25]$. The percentage of summed GC peak areas (referred to as relative abundances) of volatiles accurately reflects genetic relatedness and thus can be used to analyze the genotype-related odortypes $[1,5]$. Here, the PCA and pairwise comparison of the relative abundances of $\mathrm{BU}$ volatiles and PG volatiles suggested that the genetic relatednesscorrelated odortypes were determined by the volatile profiles with quantitative variation and/or some key urinary volatiles and thus might be used as genetic compatibility criteria in these rats.

In our previous work in rats, a few urinary volatiles, such as 2-heptanone, 4-ethyl phenol and squalene, have been identified as male pheromones, which alone or in combination can attract females and reflect genetic relatedness [5,25]. Here, since the above-mentioned 7 major BU volatiles are at higher levels in males than in females, and dependent on testis and androgen, they are male sex pheromone candidates $[2,25]$. On the other hand, all or most of these 7 compounds were consistently over-expressed in LL males, as compared to BB and hybrid males; therefore, they may function as an extravagant male trait to advertise high quality. Such conclusions were further exemplified by replenishing the ketones into $\mathrm{BB}$ and castrated male urine, which resulted 

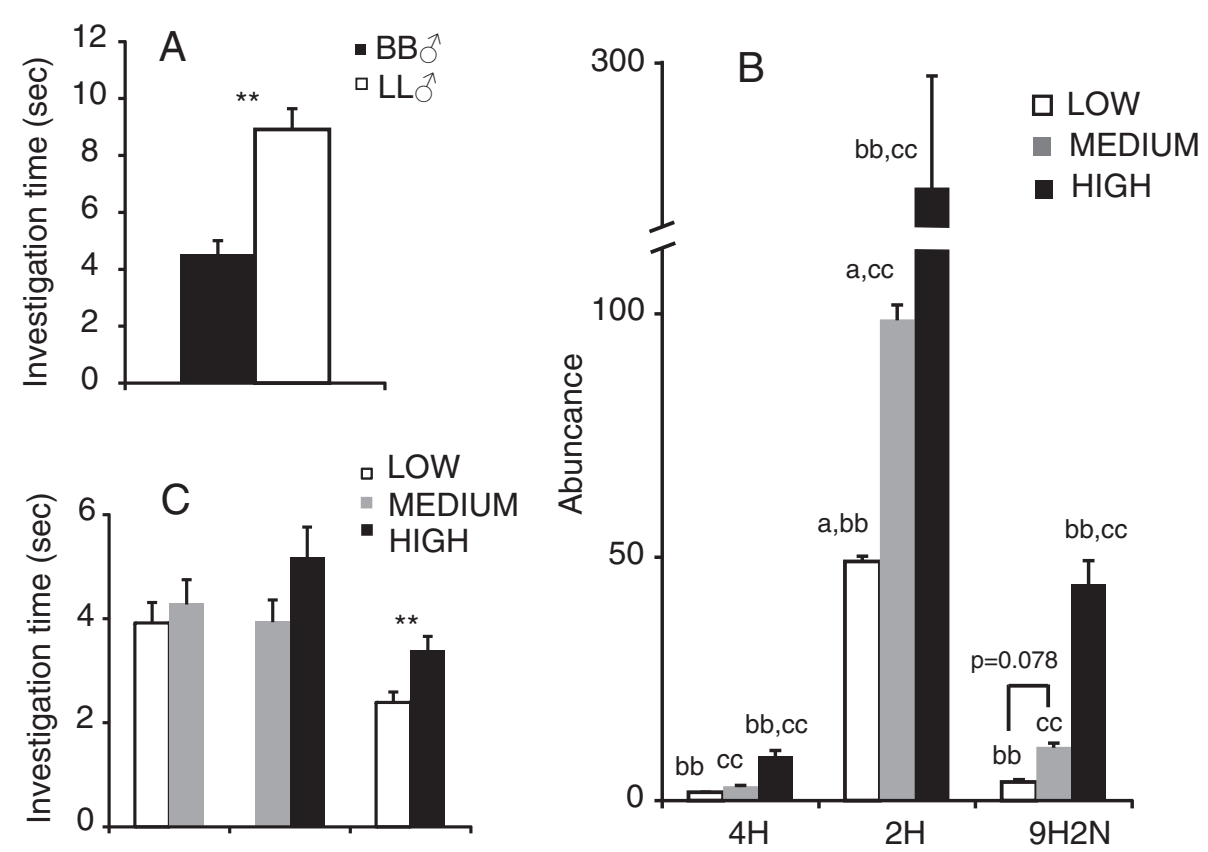

Figure 6 Behavioral and chemical data of F2 male urine. (A) Duration of investigation (mean \pm SE) by F2 BL females ( $N=40)$ of different male urine samples during a 3 -min binary choice test ${ }^{* *} P<0.01$, paired $t$-test). (B) Comparison of 3 urinary major ketones among LOW, MEDIUM and $\mathrm{HIGH}$ group of the $B L F 2$ male urine ( $N=20$, mean $\pm S E$, one way ANOVA with post hoc LSD test, the same superscript letters indicate significant difference, single letter, $P<0.05$; double letters, $P<0.01$ ). (C) Duration of investigation (mean $\pm \mathrm{SE}$ ) by F2 BL females $(N=40)$ of F2 BL male urine of LOW, MEDIUM and HIGH groups during a 3-min binary choice test ( ${ }^{*} P<0.01$, Wilcoxon signed rank test).

in equalization of the sexual attractiveness of the adjusted BB, adjusted castrated male urine and LL male urine. However, few of these pheromone components differed between BB and F1 hybrid male urine and thus LL female mate choice was majorly influenced by genetic compatibility. These results indicated that these compounds were of male pheromones and when above a certain concentration, could serve as an extravagant male trait. Similar results were also observed in genetically diverse F2 hybrids. Specifically, the F2 males of HIGH group were more strongly attractive to F2 females than those of LOW group, suggesting that extravagant male pheromones-based preference might be implicated in mate choice of wild populations.

Since the recipients could sense both volatile and non-volatile cues in male urine during our 2-choice tests, we also measured the major urine proteins (MUPs) of these strains and their F2 generation using sodium dodecyl sulfate-polyacrylamide gel electrophoresis (SDS-PAGE). We have not observed the between-strain differences of MUPs (data not shown). Such a 2-choice test method has been successfully used in our previous work [5]. Here, a close correlation was found between the volatiles and the female preferences. It implied that the female chemosensory preferences might be mainly shaped by the volatile ketone pheromones rather than MUPs [34].
Here, we first reported in rats that genetic compatibility and sexual attractiveness criteria signaled by urine-borne volatiles interact to affect odor-based female mate preferences $[4,35]$. The variation in male pheromones of potential mates allowed LL females to weigh the trade-off between genetic compatibility and quality benefits. The extravagant male pheromones in LL males served as a chemical ornament trait or high genetic quality trait to attract LL females, independent of genetic compatibility $[32,33]$. The sexual attractiveness indicated by male pheromones in LL males could consistently predict the preferences of LL females independent of genetic dissimilarity and heterozygosity, whereas genetic compatibility could influence the preferences only when male pheromones of potential mates varied on a small scale, for example, the preferences between BB and the F1 hybrid males $[2,4,12]$.

Such a male pheromone-indicating quality might be associated with "good genes" and sexy son benefits, where the "good genes" benefit focuses on the increased viability of both sons and daughters and the sexy son benefit suggests an indirect effect of the attractiveness of sons at the cost of offspring fitness [11]. Thus far, theoretical studies and a recent meta-analysis of 90 studies on 55 animal species have suggested that the choosy females get "sexy sons" more often than "good genes", but few experiments have directly demonstrated the "sexy sons" mechanism [11,12,33,36,37]. When choosing 
attractive LL males, LL females gained the attractive traits, but took the risk of a fitness reduction due to inbreeding depression, suggesting that extravagant male pheromones reflected sexy son benefits rather than "good genes" [11,32,38].

Although male pheromones in rats are under the control of androgen, we did not detect the difference of serum testosterone levels between $\mathrm{BB}$ and LL males $(P=0.482$, $Z=0.703, N=7$ ) [39]. Therefore, the "extravagant chemical ornament" highly expressed in homozygous LL males, might be determined by polygenes from both homozygous LL parents. Mating between relatives can spread genes identical by descent, which offers a positive effect on the inclusive fitness of parents $[12,14,16,17,38,40]$. The decreased fitness and the increased expression of deleterious recessive genes in the offspring would offset the benefits of sexual attractiveness for mating success [32]. The male pheromones acting as attractive traits were suppressed in BB males but highly expressed in LL males, suggesting that male pheromones and sexual attractiveness are not always reduced by inbreeding [41,42]. Most theoretical and experimental studies assume that the genes regulating emission and perception of sensory signals are separate but linked, except in the case of two studies in the medaka (Oryzias latipes) and the fruit fly (Drosophila melanogaster) showing a single-gene control of both pheromone emission and perception $[43,44]$.

\section{Conclusions}

We suggest that the exaggerated male pheromones produce a "sexual chemical ornament" that consistently attracts females, independent of genetic compatibility. Genetic compatibility influenced the female preferences only when the male pheromones of potential mates varied on a small scale. Despite the risk of inbreeding, choosy females might gain the advantages of homozygous sexy sons by mating with relatives. The volatile signals can transmit information at a distance, and precede the first episode of mating behavior and consequently increased the potential reproductive success $[34,45]$. The male "chemical ornament" and "sexy genes" benefits might also give an additional explanation for inclusive fitness with inbreeding in natural population $[11,22]$.

\section{Methods}

\section{Subject}

In experiment $1, \mathrm{BN}$ rats ( $\mathrm{BB}, N=16$, half of each sex) and Lewis rats (LL, $N=16$, half of each sex) at 12 weeks of age, obtained from Vital River Laboratory Animal Technology Co. Ltd, were reciprocally crossed to produce $\mathrm{F} 1$ hybrid rats $\left(\mathrm{BL}=\mathrm{BB}+\times \mathrm{LL}^{\dagger} ; \mathrm{LB}=\mathrm{LL}+\times \mathrm{BB}^{\hat{}}\right) . \mathrm{BB}$ $(N=20$, half of each sex) and LL $(N=16$, half of each sex $)$ were used to obtain pure BB and LL F1 rats. $15 \mathrm{BL}$ males were bred with $30 \mathrm{BL}$ females to produce BL F2 rats. The males were removed when the females had become noticeably pregnant. The offspring were weaned, weighed, sexed at 4 weeks, and then housed with same sexed siblings (4-6 per cage).

In experiment 2, 21 LL rats (12 weeks old; Vital River Laboratory Animal Technology Co. Ltd) were assigned to the following treatment groups: bilateral orchiectomy $(N=7)$; sham orchiectomy $(N=7)$; bilateral orchiectomy with testosterone supplement $(N=7)$. In the orchiectomy group, rats were anesthetized with sodium pentobarbital (45 mg/kg), and then the testes were excised following incision of the scrotum and ligation of the blood vessels and vas deferens. The sham orchiectomy procedure was the same, but the testes were not excised. A silastic capsule (1.57 mm inner diameter, $20 \mathrm{~mm}$ length, left blank or filled with testosterone, incubated in $0.9 \%$ sodium chloride solutions for $48 \mathrm{~h}$ ) was implanted subcutaneously between the scapulae of each rat. Rats were single-housed for a 4-week postsurgical recovery period, and then served as urine and PGS donor.

In addition, $40 \mathrm{BB}$ females, $60 \mathrm{LL}$ females and $100 \mathrm{BL}$ females (some of them brought from Vital River Laboratory Animal Technology Co. Ltd and some of them F1 and F2 offspring from the present experiment) were used as odor recipients in binary choice tests. Urine collection, binary choice test and chemical analysis were carried out at 12-16 weeks. The room had a reversed 14:10 h light: dark photoperiod (lights on at 19:00) and was maintained at $23 \pm 2^{\circ} \mathrm{C}$. Rats were kept in rat cages $(37 \times 26 \times 17 \mathrm{~cm})$. Food (standard rat chow) and water were provided ad libitum.

\section{Scent collection and sample preparation}

Each rat was placed in a clean cage $(25 \times 15 \times 13.5 \mathrm{~cm}$, fitted with a wire grid $1 \mathrm{~cm}$ above the cage floor), and urine was collected immediately and transferred to a sealed glass vial on ice. Urine contaminated by feces was discarded. Rats were decapitated, and the paired preputial glands were immediately dissected and stored in vials. Urine and preputial glands were kept at $-20^{\circ} \mathrm{C}$ prior to analysis.

To prepare urine samples treated with the identified synthetic analogs for behavioural assays, 4-heptanone, 2-heptanone and 9-hydroxy-2-nonanone were dissolved in dichloromethane. The solution is added in to a vial and been vaporized for 3 minutes before adding urine. Meanwhile, equal amount of pure dichloromethane were added into urine controls for comparison with fortified urines. Male urine was then mixed equally from 7 urine donors, and added to the vial.

To characterize the composition of urine samples, we mixed $120 \mu \mathrm{L}$ dichloromethane (purity >99.5\%; DIMA Technology, Inc.) with $120 \mu \mathrm{L}$ urine, stirred the mixture thoroughly, stored it at $4^{\circ} \mathrm{C}$ for $12 \mathrm{~h}$, and then used the 
bottom phase (the layer with dichloromethane) for chemical analysis.

\section{Two-choice olfactory tests}

We determined the estrus cycles of female rats, and used the estrus ones as odor recipients. Double blind binary choice tests were carried out under dim red light during the dark phase of the light cycle. For each test, we left one subject in its home cage while temporarily removed its cage mates. We painted $2 \mu \mathrm{L}$ of urine samples on one end of a glass rod $(20 \mathrm{~cm}$ long, $4 \mathrm{~mm}$ diameter) and held the other end with plastic gloves. Two scented glass rods were poked through the bars of the cage, and simultaneously presented to the subject. The two ends painted with urine samples were $1.5 \mathrm{~cm}$ apart from each other. The investigation time for each urine sample was recorded for $3 \mathrm{~min}$ after the subject first sniffed or licked the rod tip. The experimenter was blind to the nature of the sample. To control for experimenter bias, we exchange the positions of odor samples when testing the next female. Recipients were randomly chosen, and each rat was used only once a day. Each female was used once in a day and given at least 4 days of rest before another test. Each subject was used in one or two tests. The tests of BB and LL females were replicated once. As many of the subjects were only tested once, we did not assess the random effects and subject variations. Recipients were allowed to freely investigate the scented glass rods in their home cage, and those whose investigating time was less than $1 \mathrm{~s}$ were excluded for the day.

Gas chromatography- mass spectrometry (GC-MS)/GC assay Analysis of F1 urine and PGS were performed on an Agilent 6890 N GC System connected to the 5973 Mass Selective Detector (NIST 2002 Library; Agilent Technologies, Inc., USA). The GC was equipped with a non-polar column (HP5MS, $30 \mathrm{~m}$ long, $0.25 \mathrm{~mm}$ inner diameter, $0.25 \mu \mathrm{m}$ film) as well as a polar column (DBWAX, $30 \mathrm{~m}$ long, $0.25 \mathrm{~mm}$ inner diameter, $0.25 \mu \mathrm{m}$ film). The carrier gas was Helium $(1.0 \mathrm{ml} / \mathrm{min})$. The injector temperature was set at $280^{\circ} \mathrm{C}$. The oven temperature was set initially at $50^{\circ} \mathrm{C}$, heated by $5^{\circ} \mathrm{C} / \mathrm{min}$ to $100^{\circ} \mathrm{C}$, then ramped by $10^{\circ} \mathrm{C} / \mathrm{min}$ to $280^{\circ} \mathrm{C}$, and held for $5 \mathrm{~min}$. Electron impact ionization was at $70 \mathrm{eV}$. The transfer line temperature was set at $280^{\circ} \mathrm{C}$. Scanning mass ranged from 30 to $450 \mathrm{amu}$. We injected a $4 \mu \mathrm{L}$ sample at a splitless mode for urine and $3 \mu \mathrm{L}$ sample in a split mode (1:10) for PGS. The GC-FID and GC-MSD system are checked daily with calibration standards before running samples.

Tentative identifications were done by comparison of results on both polar and non-polar column and comparing the mass spectra of GC peaks with those in the MS library and the published literature [25,46]. The diagnostic fragments at $\mathrm{m} / \mathrm{z} 60, \mathrm{~m} / \mathrm{z} \mathrm{M}^{+}-29$, and $\mathrm{m} / \mathrm{z}$
$\mathrm{M}^{+}-43$ imply fatty acids; and $\mathrm{m} / \mathrm{z} 58, \mathrm{~m} / \mathrm{z} 71$ imply ketones. Fourteen compounds, 4-heptanone, 2-heptanone, dimethyl sulfone, 4-methyl phenol, 4-ethyl phenol, 9hydroxy-2-nonanone, indole, E- $\beta$-farnesene, dodecanoic acid, tetradecanoic acid, hexadecanoic acid, Z9-octadecenoic acid, octadecanoic acid, and squalene (all purity $>98 \%$; ACROS Organics) were further confirmed by matching retention times and mass spectra with the authentic analogs.

Analysis of BL F2 urine was performed on an Agilent 7890 N GC System connected to a flame ionization detector (FID) (Agilent Technologies, Inc., USA). The GC was equipped with a HP5-MS column, and the carrier gas was $\mathrm{N}_{2}(1.0 \mathrm{ml} / \mathrm{min})$. The inject volume, inject temperature and oven temperature were the same as those of 6890 N GC System. We compared the retention time of an F2 urine sample with the retention times of 4-heptanone, 2-heptanone and 9-hydroxy-2-nonanone authentic analogs to identify the 3 ketones. Acoording to the chemical data, we sorted the $140 \mathrm{BL}$ F2 by according to 2-heptanone levels, and chose the LOW, MEDIUM and HIGH group ( $N=20$ for each group) for the behaviour tests (mean levels of 2-heptanone: $\mathrm{HIGH}=20.33 \mathrm{ppm}$, MEDIUM = 7.15 ppm, LOW = 3.41 ppm).

We quantified 4-heptanone, 2-heptanone and 9hydroxy-2-nonanone in urine by comparing their GC areas in the samples with an established standard curve (GC area vs. concentration). The abundance and relative abundances of compounds were used for statistical analysis. For a particular compound, the abundance was quantified by GC peak area, and the relative abundance was a percentage of the summed peak areas from all targeted GC peaks.

\section{Statistical analysis}

The distribution of raw data was examined using a Kolmogorov-Smirnov test and then either parametric or nonparametric tests were used in the analyses that followed. A one-way ANOVA was applied to determine the effects of strain (in experiment 1) and hormone status (in experiment 2) on each volatile compound. One way ANOVA was followed by least significant different (LSD) tests. Independent $t$ - tests or Mann Whitney $U$ tests were used to determine the differences in concentrations of volatiles between LL male and female. Also, a principal component analysis (PCA) was applied to the chemical data to investigate whether the whole volatile profiles were differentiated between groups. The raw data were deduced, and new variables (PCs) were generated. The first two most powerful PCs, accounting for more than $60 \%$ of the total variance were plotted in two-dimensional graphs. Paired $t$ - tests or Wilcoxon signed-rank tests were applied to the binary choice data. All Statistical analysis was conducted using SPSS (v15.0, SPSS Inc.). Alpha was set at $P<0.05$. 


\section{Ethical standards}

The procedures of animal care and use in this study fully complied with Chinese legal requirements and were approved by the Animal Use Committee of the Institute of Zoology, Chinese Academy of Sciences (approval number IOZ12017).

\section{Additional files}

Additional file 1: Table S1. Breeding pairs and offspring; Table S2. The relative abundances of urinary components of 4 strains of rats; Table S3. The relative abundances of PGS components of 4 strains of rats; Table S4. The abundances of urinary components of 4 strains of rats; Table S5. The abundances of PGS components of 4 strains of rats; Table S6. The relative abundances of urinary components of male rats under different hormonal status; Table S7. The abundances of urinary components of male rats under different hormonal status.

Additional file 2: Figure S1. Representative GC profiles of urinary volatiles detected from BB, LL, BL, LB males (Numbered GC peaks correspond to the compounds in Table S2 and S4. 4H: 4-Heptanone, 2H: 2-Heptanone, 6M5H2N: 6-Methyl- 5-hepten-2-one, 3E2,4HD: 3-Ethyl-2, 4-heptanedione, DTHP2N: a dialkyl tetrahydro-2H-pyran-2-one, 9H2N: 9-hydroxy-2-Nonanone).

Additional file 3: Figure S2. GC chromatogram of PGS volatiles detected from BB males (upper panel) and LL males (lower panel) (Numbered GC peaks correspond to the compounds in Table S3 and S5).

Additional file 4: Figure S3. Principal component plots of $B B, L L$ and hybrid males, based on the relative abundances $(A)$ and absolute abundances (B) of the BU volatiles. Each symbol represents 1 individual rat, and the percentage of the total variance along each principal component is given.

Additional file 5: Figure S4. Principal component plots of $B B$ and $L L$ males, based on the relative abundances: $(A)$ and absolute abundances (B) of the PGS volatiles. Each symbol represents 1 individual rat, and the percentage of the total variance along each principal component is given.

Additional file 6: Figure S5. Comparison of 7 urinary major ketones between male and female $L L$ rats: $(A)$ abundances, $(B)$ relative abundances. (mean $\pm \mathrm{SE}$, single asterisk, $P<0.05$; double asterisks, $P<0.01$. 4H: 4-Heptanone, 2H: 2-Heptanone, 6M5H2N: 6-Methyl- 5hepten-2-one, 3E2,4HD: 3-Ethyl-2,4-heptanedione, DTHP2N: a dialkyl tetrahydro-2H-pyran-2-one, 9H2N: 9-hydroxy-2-Nonanone).

Additional file 7: Figure S6. Duration of investigation (mean $\pm \mathrm{SE}$ ) by female $L L$ rats $(N=13)$ of different male urine samples during a 3-min binary choice test: castrated male urine vs. castrated male urine added with 4-heptanone, $(4 \mathrm{H}, 0.5$ ppm) , 2-heptanone (2H, 4 ppm) or 9-hydro-2nonanoe $(9 \mathrm{H} 2 \mathrm{~N}, 9.6 \mathrm{ppm})$ at minimal effective concentrations, respectively ( ${ }^{*} P<0.05,{ }^{* *} P<0.01$, paired $t$-test).

\section{Competing interests}

The authors declare that they have no competing interests.

\section{Authors' contributions}

JXZ and YHZ designed the study. YHZ and JXZ wrote the paper. YHZ collected and analyzed the data. Both authors read and approved the final manuscript.

\section{Acknowledgments}

This work was supported by the Strategic Priority Research Program of the Chinese Academy of Sciences [XDB1 1010400], the grants from China National Science Foundation [91231107 and 31301887], Chinese Academy of Sciences [KSCX2-EW-N-5], and the Foundation of State Key Laboratory of IPM [ChineselPM1303]. We are most grateful for Mr. Jin-Hua Zhang's assistance with behavioral tests and Prof. Peter Keightley' s help with improving the manuscript.
Received: 5 June 2014 Accepted: 25 September 2014

Published online: 30 October 2014

\section{References}

1. Singer AG, Beauchamp GK, Yamazaki K: Volatile signals of the major histocompatibility complex in male mouse urine. Proc Natl Acad Sci USA 1997, 94:2210-2214.

2. Novotny M, Harvey S, Jemiolo B: Chemistry of male dominance in the house mouse, mus domesticus. Experientia 1990, 46:109-113.

3. Penn D, Potts W: MHC-disassortative mating preferences reversed by cross-fostering. Proc R SOC B 1998, 265:1299-1306.

4. Roberts SC, Gosling LM: Genetic similarity and quality interact in mate choice decisions by female mice. Nat Genet 2003, 35:103-106.

5. Zhang $\mathrm{YH}$, Zhang JX: Urine-derived key volatiles may signal genetic relatedness in male rats. Chem Senses 2011, 36:125-135.

6. Tregenza T, Wedell N: Genetic compatibility, mate choice and patterns of parentage: Invited review. Mol Ecol 2000, 9:1013-1027.

7. Thünken T, Meuthen D, Bakker TCM, Baldauf SA: A sex-specific trade-off between mating preferences for genetic compatibility and body size in a cichlid fish with mutual mate choice. Proc R SOC B 2012, 279:2959-2964.

8. Cheetham SA, Thom MD, Jury F, Ollier WER, Beynon RJ, Hurst JL: The genetic basis of individual-recognition signals in the mouse. Curr Biol 2007, 17:1771-1777.

9. Sherborne AL, Thom MD, Paterson S, Jury F, Ollier WE, Stockley P, Beynon $R J$, Hurst $J$ : The genetic basis of inbreeding avoidance in house mice. Curr Biol 2007, 17:2061-2066.

10. Thom MD, Stockley P, Jury F, Ollier WER, Beynon RJ, Hurst JL: The direct assessment of genetic heterozygosity through scent in the mouse. Curr Biol 2008, 18:619-623.

11. Andersson M, Simmons LW: Sexual selection and mate choice. Trends Ecol Evol 2006, 21:296-302.

12. Neff BD, Pitcher TE: Genetic quality and sexual selection: an integrated framework for good genes and compatible genes. Mol Ecol 2005, 14:19-38.

13. Brown RE, Singh PB, Roser B: The major histocompatibility complex and the chemosensory recognition of individuality in rats. Physiol Behav 1987, 40:65-73.

14. Eizaguirre C, Yeates SE, Lenz TL, Kalbe M, Milinski M: MHC-based mate choice combines good genes and maintenance of MHC polymorphism. Mol Ecol 2009, 18:3316-3329.

15. Head ML, Hunt J, Jennions MD, Brooks R: The indirect benefits of mating with attractive males outweigh the direct costs. PLoS Biol 2005, 3:e33.

16. Kokko H, Ots I: When not to avoid inbreeding. Evolution 2006, 60:467-475.

17. Puurtinen M: Mate Choice for Optimal (K) Inbreeding. Evolution 2011, 65:1501-1505.

18. Schjørring S, Jäger I: Incestuous mate preference by a simultaneous hermaphrodite with strong inbreeding depression. Evolution 2007, 61:423-430.

19. Thünken T, Bakker TCM, Baldauf SA, Kullmann H: Active inbreeding in a cichlid fish and its adaptive significance. Curr Biol 2007, 17:225-229.

20. Loyau A, Cornuau JH, Clobert J, Danchin E: Incestuous Sisters: Mate Preference for Brothers over Unrelated Males in Drosophila melanogaster. PLOS ONE 2012, 7:e51293.

21. Robinson SP, Kennington WJ, Simmons LW: Preference for related mates in the fruit fly, Drosophila melanogaster. Anim Behav 2012, 84:1169-1176.

22. Szulkin M, Stopher KV, Pemberton JM, Reid JM: Inbreeding avoidance, tolerance, or preference in animals? Trends Ecol Evol 2013, 28:205-211.

23. Hepper PG: The discrimination of different degrees of relatedness in the rat - evidence for a genetic identifier. Anim Behav 1987, 35:549-554.

24. Hurst JL, Payne CE, Nevison CM, Marie AD, Humphries RE, Robertson DH, Cavaggioni A, Beynon RJ: Individual recognition in mice mediated by major urinary proteins. Nature 2001, 414:631-634.

25. Zhang JX, Sun L, Zhang JH, Feng ZY: Sex- and gonad-affecting scent compounds and 3 male pheromones in the rat. Chem Senses 2008, 33:611-621.

26. Bender K, Balogh P, Bertrand MF, Den Bieman M, Von Deimling O, Eghtessadi S, Gutman GA, Hedrich HJ, Hunt SV, Kluge R, Matsumoto K, Moralejo DH, Nagel M, Portal A, Prokop C-M, Seibert RT, van Zutphen LFM: Genetic characterization of inbred strains of the rat (Rattus norvegicus). J Exp Anim Sci 1994, 36:151-165. 
27. Isles AR, Baum MJ, Ma D, Keverne EB, Allen ND: Genetic imprinting - Urinary odour preferences in mice. Nature 2001, 409:783-784.

28. Hopp SL, Owren MJ, Marion JR: Olfactory discrimination of individual littermates in rats (Rattus norvegicus). J Comp Psychol 1985, 99:248-251.

29. Wyatt TD: Pheromones and signature mixtures: defining species-wide signals and variable cues for identity in both invertebrates and vertebrates. J Comp Physiol Sensory Neural Behav Physiol 2010, 196:685-700.

30. Johnston RE: Chemical communication in rodents: from pheromones to individual recognition. J Mammal 2003, 84:1141-1162.

31. Gabirot M, Lopez P, Martin J: Female mate choice based on pheromone content may inhibit reproductive isolation between distinct populations of Iberian wall lizards. Curr Zool 2013, 59:210-220.

32. Brooks R: Negative genetic correlation between male sexual attractiveness and survival. Nature 2000, 406:67-70.

33. Huk T, Winkel WG: Testing the sexy son hypothesis - a research framework for empirical approaches. Behav Ecol 2008, 19:456-461.

34. Brennan PA, Kendrick KM: Mammalian social odours: attraction and individual recognition. Philos Trans R Soc Lond B Biol Sci 2006, 361:2061-2078.

35. Kokko H, Brooks R, McNamara JM, Houston Al: The sexual selection continuum. Proc R Soc B 2002, 269:1331-1340.

36. Sharma MD, Griffin RM, Hollis J, Tregenza T, Hosken DJ: Reinvestigating good genes benefits of mate choice in drosophila simulans. Biol I Linn Soc 2012, 106:295-306

37. Prokop ZM, Michalczyk L, Drobniak SM, Herdegen M, Radwan J: Metaanalysis suggests choosy females get sexy sons more than "good genes". Evolution 2012, 66:2665-2673.

38. Taylor ML, Wedell N, Hosken DJ: The heritability of attractiveness. Curr Biol 2007, 17:R959-R960.

39. Taylor GT, Haller J, Regan D: Female Rats Prefer an Area Vacated by a High Testosterone Male. Physiol Behav 1982, 28:953-958.

40. Wedell N, Tregenza T: Successful fathers sire successful sons. Evolution 1999, 53:620-625.

41. Bolund E, Martin K, Kempenaers B, Forstmeier W: Inbreeding depression of sexually selected traits and attractiveness in the zebra finch. Anim Behav 2010, 79:947-955.

42. Van Bergen E, Brakefield PM, Heuskin S, Zwaan BJ, Nieberding CM: The scent of inbreeding: a male sex pheromone betrays inbred males. Proc R Soc B 2013, 280:20130102.

43. Fukamachi S, Kinoshita M, Aizawa K, Oda S, Meyer A, Mitani H: Dual control by a single gene of secondary sexual characters and mating preferences in medaka. BMC Biol 2009, 7:64.

44. Bousquet F, Nojima T, Houot B, Chauvel I, Chaudy S, Dupas S, Yamamoto D, Ferveur JF: Expression of a desaturase gene, desat1, in neural and nonneural tissues separately affects perception and emission of sex pheromones in Drosophila. Proc Natl Acad Sci USA 2012, 109:249-254

45. Thonhauser KE, Raveh S, Hettyey A, Beissmann H, Penn DJ: Scent marking increases male reproductive success in wild house mice. Anim Behav 2013, 86:1013-1021.

46. Zhang JX, Rao XP, Sun $L X$, Zhao CH, Qin XW: Putative chemical signals about sex, individuality, and genetic background in the preputial gland and urine of the house mouse (Mus musculus). Chem Senses 2007, 32:293-303.

\section{doi:10.1186/s12983-014-0073-x}

Cite this article as: Zhang and Zhang: A male pheromone-mediated trade-off between female preferences for genetic compatibility and sexual attractiveness in rats. Frontiers in Zoology 2014 11:73.

\section{Submit your next manuscript to BioMed Central and take full advantage of:}

- Convenient online submission

- Thorough peer review

- No space constraints or color figure charges

- Immediate publication on acceptance

- Inclusion in PubMed, CAS, Scopus and Google Scholar

- Research which is freely available for redistribution

Submit your manuscript at www.biomedcentral.com/submit
Biomed Central 